\title{
It all sounds the same to me: Sequential ERP and behavioral effects during pitch and harmonicity judgments
}

\author{
BENJAMIN J. DYSON \\ Ryerson University, Toronto, Ontario, Canada \\ AND \\ Claude Alain \\ Baycrest Centre for Geriatric Care, Toronto, Ontario, Canada \\ and University of Toronto, Ontario, Canada
}

\begin{abstract}
The representation of complex sounds was examined by comparing both behavioral and event-related brain potentials (ERPs) to the change or repetition of fundamental frequency $\left(f_{0}\right)$ and harmonicity. In the pitch task, participants were asked to categorize the incoming stimulus as either low or high, regardless of harmonicity, and in the harmonicity task, participants indicated whether the stimulus was tuned or mistuned, regardless of pitch. Over three experiments, participants were faster in responding to pitch than to harmonicity. As a result of this asymmetry, behavioral and ERP data showed that irrelevant changes in harmonicity had little impact on performance during the pitch task, whereas harmonicity judgments were impeded by irrelevant changes in $f_{0}$. These data are consistent with both general horse-race accounts of processing and specific accounts of mistuning detection that posit prior $f_{0}$ registration. In addition, ERP components $\mathrm{N} 2$ and $\mathrm{P} 3$ were modulated by both intertrial contingency and task instructions, revealing the further influence of top-down mechanisms on concurrent sound segregation.
\end{abstract}

The perception of complex auditory events requires an individual to group and separate incoming acoustic data into meaningful events (see Dyson, in press, for a review). Specifically, according to Bregman's (1990) two-stage account of auditory scene analysis, frequency periodicity (i.e., harmonicity) acts in conjunction with several other cues, such as onset-offset and common location, to assist in the primary segregation of acoustic energy. Once this input has initially been partitioned, the auditory system is able to implement more controlled processes in order to arrive at individual representations of objects or streams. The expectation is that the initial segregation of acoustic energy should be automatic and not contingent upon attention (Bregman, 1990; although see Cusack, Deeks, Aikman, \& Carlyon, 2004; Reinke, He, Wang, \& Alain, 2003), whereas the formation of higher order representations should be more reliant upon the use of top-down (or schema-based) processing.

One type of investigation that has proved useful in studying the representation of concurrent sound objects is the mistuned harmonic paradigm. Here, participants are typically played complex auditory stimuli, built up from a number of pure tone components, and are asked to make certain kinds of psychoacoustic judgments relating to the sounds (such as pitch matching; e.g., Darwin, Hukin, \&
Al-Khatib, 1995). A critical distinction is made between tuned stimuli, in which all tonal components are integer multiples of the fundamental frequency $\left(f_{0}\right)$, and mistuned stimuli, in which one partial is not an integer multiple of the $f_{0}$. Under the appropriate conditions, mistuning can promote concurrent sound perception in that the mistuned partial perceptually "pops out" of the complex sound, in much the same way as a visual target is defined by unique color "pop-outs" of a display filled with homogeneous distractors defined by a secondary color (Wolfe, 1998). The phenomenological experience associated with the harmonicity of a complex is usually a "buzz" for the tuned stimulus, and a "buzz" with a separate pure tone element for the mistuned stimulus (Moore, Glasberg, \& Peters, 1986). In the latter case, the "buzz" represents all harmonically related partials, and the pure tone component represents the inharmonic partial. Therefore, the mistuned harmonic paradigm can be used to distinguish between cases of singular and multiple concurrent objects within an auditory scene, with the tuned stimulus representing one object and the mistuned stimulus representing two (Alain, Arnott, \& Picton, 2001; Alain, Schuler, \& McDonald, 2002; Dyson \& Alain, 2004).

Cognitive models put forward to account for the perception of concurrent sound objects posit the use of a har- 
monic sieve or template (Duifhuis, Willems, \& Sluyter, 1982; Lin \& Hartmann, 1998; Scheffers, 1983; but see Roberts \& Holmes, 2006, for a brief discussion of potential temporal contributions to the perception of mistuning). The formation of this template implies a rapid estimation of the dominant $f_{0}$, which depends primarily on neurons sensitive to frequency periodicity observed in the acoustic wave (see Alain, 2007, p. 227). For example, if the $f_{0}$ is estimated at $200 \mathrm{~Hz}$, the auditory system becomes sensitive to 400, 600, and $800 \mathrm{~Hz}$, allowing harmonically related partials to group together with the $f_{0}$, whereas those acoustic elements that fail to share the approximate multiple integer relationship (such as the mistuned partial) form separate representations.

One of the issues with the template matching model described above is that it does not take into account the role of previous auditory experience in shaping the representation of harmonic templates. This seems particularly important, since researchers have demonstrated that certain aspects of harmonicity processing can be modulated by attention. For example, Darwin et al. (1995) found that the contribution of a mistuned partial in judging overall pitch could be reduced by exposing participants to a train of pure tones prior to the presentation of the complex sound. Specifically, if this series of sounds represented the frequency of the mistuned partial within the to-bejudged complex, independently of whether these sounds were presented contra- or ipsilaterally to the rest of the complex, the pitch of the complex was more accurately judged than in the absence of these sounds. Similar effects have also been observed for onset (Darwin \& Ciocca, 1992) and frequency and amplitude modulation (Darwin, Ciocca, \& Sandell, 1994). The studies by Darwin and colleagues are important in the way they emphasize the role of prior information on the organization of simultaneous acoustic input; that is, once a separate auditory stream has been established on the basis of pitch (in this case, the mistuned harmonic), the subsequent reintegration of that frequency component into a complex sound becomes less likely, as reflected in the reduced contribution that the mistuned partial makes to the pitch of the complex.

However, procedures such as those employed by Darwin et al. (1995), in which streams are formed prior to the stimulus of interest, may not be optimal to assess the joint contribution of bottom-up and top-down effects on harmonic template development and maintenance. One specific problem is that the level of acoustic history (or inattention) given to the train of pure tones prior to the presentation of the complex cannot be directly assessed, and as such we do not know whether the observed effect is due to low-level segregation mechanisms or top-down strategic effects. Instead, one particular form of intertrial repetition paradigm may yield a number of advantages when it comes to examining such effects. Generally attributed to Bertelson (1961; although see Hyman, 1953, for an earlier demonstration), repetition effects are robust aspects of choice reaction time (RT) performance (Kornblum, 1973). They can be broadly defined as the facilitation of RT when stimulus and response are repeated over successive trials, relative to the case when stimulus or response changes (Rabbitt, 1992), and may reflect the reuse of functional pathways over trials (Pashler \& Baylis, 1991); that is, putative processing modules associated with the completion of trial $n$ will remain active for a nominal time after the event. If trial $n+1$ occurs soon after trial $n$, such that activation from the previous trial has not yet decayed, processing is facilitated relative to the point at which a match is found between the previous and current stimulus. To take an example from the visual literature, a perceptual match between the re-presentation of the same letter "p" on trials $n$ and $n+1$ in a letter-number discrimination paradigm should accrue more facilitation than would a categorical letter match between the presentation of "p" at trial $n$ and "b" at trial $n+1$. Moreover, if the experimental design is such that there is no systematic benefit in the retention of stimulus and/or response information across trials, researchers have argued that the resultant data should reflect "more or less automatic intertrial carryover effects" (Olivers \& Humphreys, 2003, p. 651; see also Dyson \& Quinlan, 2004). Therefore, in contrast to paradigms that make the nature of repetition explicit, such as that of Darwin et al. (1995), data collected from equiprobable intertrial contingencies provide us with an opportunity to assess how, in the absence of strategy, harmonic templates are formed and maintained within a changing sound environment.

Clearly, the purest test of the bottom-up updating of harmonic templates is both the use of equiprobable intertrial contingency presentation and a condition in which no task is defined, no responses are required, and the participant is actively encouraged to ignore the stimuli. Experiment 3 will take advantage of the fact that auditory ERPs can be recorded under such conditions, thereby providing an explicit comparison between the processes involved in the maintenance of $f_{0}$ and harmonicity information in the absence of an explicit task, and top-down contributions according to specific task demands. To begin with, Experiments 1 and 2 will investigate the more modest comparison between two different top-down influences: the active categorization of complex sounds according to $f_{0}$ versus the active categorization of complex sounds according to harmonicity. In this respect, the present data will also inform the extent to which the processing of auditory $f_{0}$ and harmonicity information is contingent with general frameworks developed in vision, such as horse-race accounts of processing (Mordkoff \& Yantis, 1991; Mordkoff, Yantis, \& Egeth, 1990). Here, general principles of processing exist for multidimensional stimuli $(x y)$ when one attribute $(x)$ is discriminated faster than another attribute $(y)$. When the retrieval of attribute $x$ is required for task completion, task-irrelevant attribute $y$ will have a negligible effect on processing as a result of its slower processing speed. However, if attribute $y$ is task relevant, processing interference will result as a consequence of attribute $x$ being completed prior to the availability of attribute $y$. The harmonic template account of Lin and Hartmann (1998) appears then to be one specific instance of a horse-race model of processing, in which harmonicity processing is generally slower than $f_{0}$. As a result, we should observe frequency to be the faster dimension, harmonicity to be the slower dimension, 
and, consequently, frequency to interfere with harmonicity but not vice versa. However, the interaction between these acoustic dimensions is particularly interesting in this regard since, under one reading, $f_{0}$ and harmonicity may actually be a special case of the horse-race model, in which one stimulus attribute (i.e., harmonicity) is dependent on the output of a second attribute (i.e., $f_{0}$ ). Therefore, a strict interpretation of the harmonic template model would be that harmonicity judgment could never be as fast as $f_{0}$ judgments, as a result of the putative architecture that produces these outputs.

Therefore, the present experimental series sought to more clearly delineate the extent to which top-down task demands interact with the bottom-up maintenance of harmonic templates over time by varying response demands within an intertrial repetition paradigm, and the degree to which the processing of auditory dimensions, such as $f_{0}$ and harmonicity, adhere to visual horse-race accounts of processing.

\section{EXPERIMENTS 1A AND 1B}

Experiments 1A and 1B involve the presentation of identical multidimensional auditory stimuli that differ with respect to both $f_{0}$ and harmonicity across trials. In Experiment 1A, participants were asked to categorize the stimuli relative to the overall pitch of the sound and ignore variation in harmonicity. In Experiment 1B, participants indicated whether the same stimuli were tuned or mistuned, while ignoring variations in pitch. If the processing of pitch and harmonicity is limited solely to the online computation of an incoming stimulus, no significant intertrial repetition effect should be observed. Alternatively, if the coding of pitch and harmonicity are influenced by prior stimuli (Darwin \& Ciocca, 1992; Darwin et al., 1995), this should be reflected in the modulation of RT and/or error rate, as a result of the different stimulus contingencies between trials $n$ and $n+1$. More specifically, if an assessment of harmonicity is contingent upon the prior registration of $f_{0}$, as predicted by the template model of frequency periodicity (Lin \& Hartmann, 1998) and a horse-race account of processing (Mordkoff et al., 1990), we might expect variation in harmonicity to have a negligible effect on pitch judgment (Experiment 1A) but for pitch variation to have a substantial influence on listeners' ability to process harmonicity (Experiment 1B). Finally, since repetition effects rely on the temporary activation of putative pathways, we must also assume that information cannot be retained indefinitely, and so must decay over time. Therefore, by manipulating the response-stimulus interval (RSI) between trials (Kirby, 1980), it should be possible to provide an estimate of the length of time for which pitch and harmonicity information is available, in a form in which it can be used to exert an influence on future stimulus processing.

\section{Experiment 1A}

\section{Method}

Participants. Informed consent for 12 participants was obtained before the experiment was conducted, according to the guidelines set out by the University of Toronto and the Baycrest Centre for Geriatric Care (age range, $21-38$ years; mean $=28.67$ years, $S D=$ 5.47). The sample consisted of 10 females and 2 males, 11 of whom were right-handed. All participants reported normal hearing and received honoraria for their involvement.

Stimuli and Apparatus. Complex sounds comprised 10 pure tone components of equal intensity and were $200 \mathrm{msec}$ in length, including 10-msec linear onset and offset ramps (SoundEdit 16, Macromedia). The stimuli were created by the combination of $f_{0}$ $(200$ or $400 \mathrm{~Hz}$ ) and the tuning of the third harmonic (tuned at 600 or $1200 \mathrm{~Hz}$, respectively; or upward mistuned by $16 \%$ : $696 \mathrm{~Hz}$ or $1392 \mathrm{~Hz}$, respectively). All other tonal components were harmonically related to the $f_{0}$. Stimuli were routed through Harman/Kardon speakers and presented over Sennheiser HD202 headphones at approximately $75 \mathrm{~dB}$ SPL, as measured on the linear scale by an artificial ear and sound level meter (Larson-Davis System 824). Stimulus presentation and response collection were controlled by Presentation software (Neurobehavioral Systems).

Design. Experiment 1A involved participants judging the overall pitch of the sounds, irrespective of harmonicity. A total of four stimulus contingencies existed between trials $n$ and $n+1$, involving the use of same (S) or different (D) $f_{0}(\mathrm{~F})$ and harmonicity $(\mathrm{H})$ values. First, the stimulus at trial $n$ could repeat with respect to both $f_{0}$ and harmonicity value at trial $n+1$ (e.g., $200 \mathrm{~Hz}$ tuned, followed by $200 \mathrm{~Hz}$ tuned; FSHS). Second, the $f_{0}$ value could be maintained across the trial but the harmonic value could change (e.g., $200 \mathrm{~Hz}$ tuned, followed by $200 \mathrm{~Hz}$ mistuned; FSHD). Third, $f_{0}$ value could change, with the harmonicity value remaining stable across trials $n$ and $n+1$ (e.g., $200 \mathrm{~Hz}$ tuned, followed by $400 \mathrm{~Hz}$ tuned; FDHS). Fourth, the stimulus at trial $n$ could undergo complete stimulus change at trial $n+1$ with respect to both $f_{0}$ and harmonicity (e.g., $200 \mathrm{~Hz}$ tuned, followed by $400 \mathrm{~Hz}$ mistuned; FDHD). Stimulus contingencies took place over short (1,300 $\mathrm{msec})$ and long $(2,700 \mathrm{msec})$ RSIs between trials $n$ and $n+1$. This resulted in a basic set of 32 pairs of stimuli (4 stimulus tokens $\times 2 f_{0}$ modulations $\times 2$ harmonicity modulations $\times 2$ RSIs), which constituted a single block of practice trials. Two additional experimental blocks were also completed by the participants, containing 128 pairs (256 stimuli) of trials each. In all cases, trial order within each block was randomized.

Procedure. At the start of the experimental session, participants were given a brief overview of the experimental task. It was explained that a single auditory stimulus would be presented on every trial and they should indicate whether the complex stimuli possessed an overall "low" or "high" pitch quality, derived from a consideration of all tonal components. In this and all subsequent experiments, participants were encouraged to respond as fast and as accurately as possible. Each trial consisted of a black screen followed by the presentation of an auditory stimulus through headphones. After participants responded to the stimulus by pressing one of two keys, a fixation cross appeared for $500 \mathrm{msec}$, followed by another black screen for $300 \mathrm{msec}$, after which the next auditory stimulus was played. An additional time lag was placed in between trials $n$ and $n+1$ of either 500 or $1,900 \mathrm{msec}$, totaling trial $n$ to trial $n+1 \mathrm{RSI}$ as either 1,300 or $2,700 \mathrm{msec}$. Once participants had completed all practice and experimental blocks, they were thanked for their time and debriefed as to the purpose of the investigation.

Data analysis. Responses were collapsed over experimental blocks, and only trial $n+1$ responses in which both trial $n$ and trial $n+1$ responses were correct were considered in RT analyses. Errors were defined as trial pairs in which a correct response was given at trial $n$ and an incorrect response was given at trial $n+1$ (Quinlan, 1999). Errors of this nature constituted 1.02\% of scores. RT outliers were identified by the recursive procedure described by Jolicœur (1999; see also van Selst \& Jolicœur, 1994), resulting in the removal of a further $3.11 \%$ of scores. Data were collapsed across individual tokens of $f_{0}$ and harmonicity type, and error rate data were arcsine transformed before analysis (Winer, 1962). In the interest of brevity, only RT data are discussed in the Results and Discussion sections. However, it should be noted that in this and 
Table 1

Group Mean Response Times (RTs, in Milliseconds) and Standard Errors ( $S E$ s) for the Pitch Judgment Tasks During the Short and Long Response-Stimulus Intervals (RSIs) in Experiment 1A

\begin{tabular}{|c|c|c|c|c|}
\hline & RT & $S E$ & $\%$ Error & $\%$ Outlier \\
\hline \multicolumn{5}{|c|}{ Short RSI } \\
\hline \multicolumn{5}{|l|}{$f_{0}$ same } \\
\hline Harmonicity same & 398 & 24 & 0.78 & 3.45 \\
\hline Harmonicity different & 406 & 23 & 1.58 & 4.27 \\
\hline \multicolumn{5}{|l|}{$f_{0}$ different } \\
\hline Harmonicity same & 417 & 27 & 1.06 & 2.39 \\
\hline Harmonicity different & 423 & 30 & 1.31 & 2.65 \\
\hline \multicolumn{5}{|c|}{ Long RSI } \\
\hline \multicolumn{5}{|l|}{$f_{0}$ same } \\
\hline Harmonicity same & 458 & 24 & 0.52 & 2.65 \\
\hline Harmonicity different & 472 & 25 & 1.34 & 3.21 \\
\hline \multicolumn{5}{|l|}{$f_{0}$ different } \\
\hline Harmonicity same & 467 & 30 & 0.78 & 2.08 \\
\hline Harmonicity different & 458 & 26 & 0.80 & 4.18 \\
\hline
\end{tabular}

in all subsequent analyses, error rates were broadly consistent with RT, and there were no speed-error trade-offs that compromised the interpretation of the RT findings.

\section{Results}

Table 1 shows the group mean RT for the conditions of interest in Experiment 1A. A three-way repeated measures ANOVA featuring the factors of RSI (short, long), $f_{0}$ (repeat, change), and harmonicity (repeat, change) revealed a main effect for RSI $\left[F(1,11)=15.15, M S_{\mathrm{e}}=4,382.70\right.$, $p=.003]$, in addition to an interaction between RSI and $f_{0}$ that approached significance $\left[F(1,11)=4.71, M S_{\mathrm{e}}=\right.$ $139.48, p=.053]$. With the aggregate means of $401 \mathrm{msec}$ for short RSI $f_{0}$ repeat, $420 \mathrm{msec}$ for short RSI $f_{0}$ change, $465 \mathrm{msec}$ for long RSI $f_{0}$ repeat, and $462 \mathrm{msec}$ for long RSI $f_{0}$ change, Tukey's HSD test revealed that the only pairwise comparisons to reach significance $(p<.05)$ were those between short and long RSIs. A main effect of harmonicity also approached significance $[F(1,11)=$ $\left.4.72, M S_{\mathrm{e}}=139.48, p=.052\right]$, revealing that cases of harmonicity repetition were marginally faster $(435 \mathrm{msec})$ than were cases of harmonicity change $(440 \mathrm{msec})$. All other ANOVA terms in all cases failed to show trends toward significance $[F(1,11)<1.82, p>.205]$.

\section{Discussion}

The data from Experiment 1A show that when participants were required to make pitch judgments, the repetition or change of $f_{0}$ and harmonicity within complex sounds resulted in only modest RT variation. Although both effects were not statistically significant, $f_{0}$ change resulted in an 18 -msec slowing during short RSI, whereas harmonicity change resulted in a marginal $7-\mathrm{msec}$ slowing. The data remain equivocal, but the trends are broadly consistent with the work of Darwin et al. (1995), who showed that previous exposure to a relevant frequency influences the subsequent pitch judgments of complex sounds. However, in the absence of complementary data regarding harmonicity responses to the same stimuli, the relative influence of intertrial repetition on sound segregation based on $f_{0}$ and harmonicity could not be fully assessed.

\section{Experiment 1B}

\section{Method}

The design of Experiment 1B was identical to that of Experiment 1A, apart from a change in task instruction. Participants were now required to make a one-two object distinction related to the absence or presence of mistuning, respectively (cf. Alain et al., 2001). An initial sample of 12 individuals was run in Experiment 1B, although 3 were replaced due to high error rates $(23.83 \%, 16.41 \%$, and $27.73 \%$ ). The final sample consisted of 3 females and 9 males, 10 of whom were right-handed (age range, 20-38 years; mean $=29.00$, $S D=4.39$ ). Again, all reported normal hearing and all received honoraria for participation.

\section{Results}

Errors constituted $3.32 \%$ of scores, and outliers made up a further $3.18 \%$ of observations. The data from Experiment 1B (see Table 2) were analyzed in an identical way to those from Experiment 1A, with all main effects and interactions showing statistical significance, apart from the main effect of harmonicity $\left[F(1,11)=0.02, M S_{\mathrm{e}}=\right.$ $568.54, p=.901 ; F(1,11)=7.51, M S_{\mathrm{e}}=1,468.15, p<$ .001 for RSI; $F(1,11)=21.55, M S_{\mathrm{e}}=1,918.47, p=.001$ for $f_{0} ; F(1,11)=14.04, M S_{\mathrm{e}}=641.06, p=.003$ for RSI $\times f_{0}$ interaction; $F(1,11)=15.08, M S_{\mathrm{e}}=394.79$, $p=.002$ for RSI $\times$ harmonicity interaction; $F(1,11)=$ $40.25, M S_{\mathrm{e}}=2,529.50, p<.001$ for $f_{0} \times$ harmonicity interaction; and $F(1,11)=9.73, M S_{\mathrm{e}}=1,852.47, p=$ .010 for the three-way RSI $\times f_{0} \times$ harmonicity interaction]. The three-way interaction was decomposed using Tukey's HSD test, revealing that significant RT costs accrued from the change of $f_{0}$ and/or harmonicity across trials $(p<.05)$ in the short RSI condition, relative to complete stimulus repetition. The longer RSI condition showed RT equivalence for all forms of stimulus contingency, but significant slowing for cases of complete stimulus repetition (FSHS) relative to the short RSI condi-

Table 2

Group Mean Response Times (RTs, in Milliseconds) and Standard Errors ( $S E s$ ) for the Harmonicity Judgment Tasks During the Short and Long Response-Stimulus Intervals (RSIs) in Experiment 1B

\begin{tabular}{|c|c|c|c|c|}
\hline & RT & $S E$ & $\%$ Error & $\%$ Outlier \\
\hline \multicolumn{5}{|c|}{ Short RSI } \\
\hline \multicolumn{5}{|l|}{$f_{0}$ same } \\
\hline Harmonicity same & 490 & 24 & 0.82 & 3.87 \\
\hline Harmonicity different & 598 & 30 & 4.31 & 4.20 \\
\hline \multicolumn{5}{|l|}{$f_{0}$ different } \\
\hline Harmonicity same & 644 & 38 & 6.95 & 3.25 \\
\hline Harmonicity different & 567 & 32 & 1.38 & 4.49 \\
\hline \multicolumn{5}{|c|}{ Long RSI } \\
\hline \multicolumn{5}{|l|}{$f_{0}$ same } \\
\hline Harmonicity same & 575 & 25 & 1.06 & 4.81 \\
\hline Harmonicity different & 596 & 25 & 3.46 & 2.82 \\
\hline \multicolumn{5}{|l|}{$f_{0}$ different } \\
\hline Harmonicity same & 635 & 32 & 3.81 & 2.77 \\
\hline Harmonicity different & 580 & 27 & 1.59 & 3.06 \\
\hline
\end{tabular}


tion. Furthermore, with respect to harmonicity processing at short RSIs, RTs to FDHS contingencies were slower than were RTs to FDHD contingencies. This pattern of data may be understood with respect to the bypass rule (Kruger \& Shapiro, 1981), according to which complete stimulus change is more readily associated with response change and complete stimulus repetition is more readily associated with response repetition. Therefore, for FDHD cases, both the response-relevant and response-irrelevant attribute changed (consistent with the bypass rule); for FDHS cases, however, the response-relevant attribute was suggestive of response repetition, whereas the responseirrelevant attribute was suggestive of change (inconsistent with the bypass rule).

A change in task instruction generated a radically different pattern of results from those observed in Experiment $1 \mathrm{~A}$. To confirm that repetition effects differentially affected pitch and harmonicity judgments, a four-way mixed design ANOVA was carried out, with the betweensubjects factor of task (pitch, harmonicity) and the withinsubjects factors of RSI (short, long), $f_{0}$ (repeat, change), and harmonicity (repeat, change). Differential repetition effects between Experiments 1A and 1B were reflected in the presence of a statistically significant four-way interaction between task, RSI, $f_{0}$, and harmonicity $[F(1,22)=$ $\left.10.69, M S_{\mathrm{e}}=1,187.72, p=.003\right]$.

\section{Discussion}

Pitch judgments were only marginally affected by intertrial contingency (Experiment 1A), but listeners were significantly slower in judging harmonicity when there was any kind of stimulus change, including a responseirrelevant change in $f_{0}$ (Experiment 1B). These patterns of data are consistent with previous models of harmonicity (Lin \& Hartmann, 1998), in which the detection of mistuning is contingent upon $f_{0}$ extraction. Our data are also consistent with predictions from a horse-race account of processing (Mordkoff et al., 1990), in which interference is larger when the irrelevant dimension is processed faster (compare Tables 1 and 2). The data from Experiment 1B suggest that concurrent sound segregation is a dynamic process that extends beyond an analysis of the current stimulus and instead relies on a comparison between the incoming stimulus and a representation of the preceding stimulus in guiding response.

However, it remains possible that participants were relying on different residual cues for tuned and mistuned stimuli. In cases where the same mistuned stimulus was repeated over trials $n$ and $n+1$, the mistuned partial would have "popped out" at trial $n$, thereby providing a single salient frequency cue for trial $n+1$, much in the same way as a train of tones at the mistuned frequency helps segregate the inharmonic partial (Darwin et al., 1995). This contrasts with the use of multiple tonal components as trial $n+1$ cues in the case of tuned stimuli. ${ }^{1}$ Moreover, mistuning the third harmonic for the $400-\mathrm{Hz} f_{0}$ complex may have led to a sensation of acoustic roughness (Moore et al., 1986). Therefore, it is possible that a salient pitch cue was only available for the $200-\mathrm{Hz}$ mistuned case, and, as such, may have facilitated repetition effects relative to the other stimulus tokens used in both versions of Experiment 1. If so, a significant interaction between harmonicity and $f_{0}$ values should be obtained for trial $n$ to trial $n+1$ relations, with the processing time for $200-\mathrm{Hz}$ mistuned stimuli being significantly different from all other stimulus tokens. In order to test this hypothesis, Experiment 1B RT data from the four different stimulus tokens collapsed across the FSHS contingency were recovered and entered into a three-way repeated measures ANOVA with the factors of RSI (1,300 msec, 2,700 msec), $f_{0}(200 \mathrm{~Hz}, 400 \mathrm{~Hz})$, and harmonicity (tuned, mistuned). In contrast to the prediction, the data revealed a main effect of RSI only $[F(1,11)=$ $\left.35.65, M S_{\mathrm{e}}=6,015.75, p<.001\right]$, with participants being slower in the long RSI case; all other main effects and interactions were nonsignificant $(p s<.094)$. The failure of harmonicity type to impact the RT data suggests that both the tuned and mistuned partials contributed to the intertrial repetition effects observed in Experiment 1B. Moreover, the absence of an interaction between $f_{0}$ and harmonicity is consistent with the notion that the putative mechanisms underlying the detection of mistuning in both the 200- and $400-\mathrm{Hz}$ complexes were similar.

\section{EXPERIMENTS 2A AND 2B}

In Experiments 2A and 2B, we tested whether the intertrial repetition effects shown in Experiment 1 may have been the result of using complex sounds that overlapped with respect to certain critical portions of the harmonic template. Indeed, every second partial was shared between the two $f_{0}$ s (i.e., $400,800,1200$, and $1600 \mathrm{~Hz}$ ). More importantly, one of these frequencies $(1200 \mathrm{~Hz})$ was the noncritical sixth harmonic in the $200-\mathrm{Hz} f_{0}$ case and the critical third harmonic in the $400-\mathrm{Hz} f_{0}$ case. Therefore, in Experiments $2 \mathrm{~A}$ and $2 \mathrm{~B}$ we used two $f_{0}$ s that were not harmonically related. Lower $f_{0} \mathrm{~s}$ were also used in order to promote the "popout" of the mistuned partial (Hartmann, 1988). Finally, a within-subjects design was implemented, thereby allowing for a more controlled comparison between the influences of intertrial contingency on pitch and harmonicity processing. If the alignment of the data with models of mistuning detection and horse-race models is independent of the specific stimuli used in Experiments 1A and 1B, the quicker registration of pitch should still interfere to a greater degree with the slower harmonicity judgment.

\section{Method}

Experiments 2A and 2B were identical to Experiments $1 \mathrm{~A}$ and $1 \mathrm{~B}$, respectively, apart from a change in the $f_{0}$ of the stimuli used- 115 and $247 \mathrm{~Hz}$. Consequently, the critical values of the tuned and mistuned third harmonic ( 345 and $397 \mathrm{~Hz}$, respectively, for the $115 f_{0}$, and 741 and $860 \mathrm{~Hz}$, respectively, for the $247 f_{0}$ ) were not shared across harmonic templates. Over two counterbalanced sessions, participants were instructed to respond (1) whether the complex sounds were globally low or high in pitch, and (2) whether the complex sounds were perceived more like one sound (i.e., tuned) or more like two sounds (i.e., mistuned). Twelve individuals participated in Experiments $2 \mathrm{~A}$ and $2 \mathrm{~B}$, although 1 participant was replaced due to a large number of RTs over $2 \mathrm{sec}$. The final sample consisted of 6 females and 6 males, 10 of whom were right-handed (age range, $23-35$ years; mean $=28.33, S D=3.23$ ). Again, all reported normal hearing and all received honoraria. 
Table 3

Group Mean Response Times (RTs, in Milliseconds) and Standard Errors ( $S E$ s) for the Pitch Judgment Tasks During the Short and Long Response-Stimulus Intervals (RSIs) in Experiment 2A

\begin{tabular}{|c|c|c|c|c|}
\hline & RT & $S E$ & $\%$ Error & $\%$ Outlier \\
\hline \multicolumn{5}{|c|}{ Short RSI } \\
\hline \multicolumn{5}{|l|}{$f_{0}$ same } \\
\hline Harmonicity same & 456 & 46 & 1.30 & 1.05 \\
\hline Harmonicity different & 469 & 49 & 2.72 & 3.26 \\
\hline \multicolumn{5}{|l|}{$f_{0}$ different } \\
\hline Harmonicity same & 489 & 55 & 0.52 & 2.61 \\
\hline Harmonicity different & 499 & 58 & 1.34 & 2.68 \\
\hline \multicolumn{5}{|c|}{ Long RSI } \\
\hline \multicolumn{5}{|l|}{$f_{0}$ same } \\
\hline Harmonicity same & 519 & 53 & 0.79 & 1.83 \\
\hline Harmonicity different & 552 & 63 & 2.97 & 2.98 \\
\hline \multicolumn{5}{|l|}{$f_{0}$ different } \\
\hline Harmonicity same & 527 & 53 & 2.13 & 2.72 \\
\hline Harmonicity different & 510 & 41 & 0.52 & 4.52 \\
\hline
\end{tabular}

\section{Results}

Of scores, $1.54 \%$ were defined as errors, whereas a further $2.71 \%$ were identified as outliers in Experiment $2 \mathrm{~A}$ (pitch judgments); the corresponding values for Experiment 2B (harmonicity judgments) were $2.67 \%$ and $3.49 \%$. Tables 3 and 4 show the RT and error rate data for the pitch and harmonicity tasks, respectively. RT data were entered into a four-way repeated measures ANOVA using the factors of task (pitch, harmonicity), RSI (short, long), $f_{0}$ (repeat, change), and harmonicity (repeat, change). All main effects were significant $\left[F(1,11)=16.83, M S_{\mathrm{e}}=58,534.55, p=\right.$ .002 for task; $F(1,11)=17.37, M S_{\mathrm{e}}=2,837.86, p=.002$ for RSI; $F(1,11)=20.82, M S_{\mathrm{e}}=3,226.36, p=.001$ for $f_{0}$, and $F(1,11)=24.01, M S_{\mathrm{e}}=1,226.49, p<.001$ for harmonicity]. The most critical interactions were the $f_{0} \times$ harmonicity interaction $\left[F(1,11)=35.59, M S_{\mathrm{e}}=2,676.84\right.$, $p<.001]$, subsumed in a larger three-way task $\times f_{0} \times$ harmonicity interaction $\left[F(1,11)=33.20, M S_{\mathrm{e}}=1,455.92\right.$, $p<.001$ ] . The aggregate means for $f_{0}$ responding (Experiment 2A) were $488 \mathrm{msec}$ for FSHS, $508 \mathrm{msec}$ for FSHD, $510 \mathrm{msec}$ for FDHS, and $505 \mathrm{msec}$ for FDHD. The aggre-

Table 4

Group Mean Response Times (RTs, in Milliseconds) and Standard Errors ( $S E$ s) for the Harmonicity Judgment Tasks During the Short and Long Response-Stimulus Intervals (RSIs) in Experiment 2B

\begin{tabular}{|c|c|c|c|c|}
\hline & RT & $S E$ & $\%$ Error & $\%$ Outlier \\
\hline \multicolumn{5}{|c|}{ Short RSI } \\
\hline \multicolumn{5}{|l|}{$f_{0}$ same } \\
\hline Harmonicity same & 528 & 25 & 1.04 & 3.00 \\
\hline Harmonicity different & 644 & 33 & 3.35 & 2.13 \\
\hline \multicolumn{5}{|l|}{$f_{0}$ different } \\
\hline Harmonicity same & 722 & 40 & 4.30 & 3.51 \\
\hline Harmonicity different & 660 & 32 & 3.73 & 2.83 \\
\hline \multicolumn{5}{|c|}{ Long RSI } \\
\hline \multicolumn{5}{|l|}{$f_{0}$ same } \\
\hline Harmonicity same & 580 & 24 & 1.15 & 4.28 \\
\hline Harmonicity different & 696 & 41 & 1.61 & 3.49 \\
\hline \multicolumn{5}{|l|}{$f_{0}$ different } \\
\hline Harmonicity same & 674 & 27 & 4.10 & 4.48 \\
\hline Harmonicity different & 663 & 26 & 2.10 & 4.17 \\
\hline
\end{tabular}

gate means for harmonicity responding (Experiment 2B) were $554 \mathrm{msec}$ for FSHS, $670 \mathrm{msec}$ for FSHD, $698 \mathrm{msec}$ for FDHS, and $661 \mathrm{msec}$ for FDHD. Tukey's HSD test $(p<$ $.05)$ revealed the absence of significant effects of intertrial contingency in Experiment 2A, significantly longer RTs in Experiment 2B, and substantial RT costs for any kind of stimulus change relative to cases of complete stimulus repetition (FSHS) during harmonicity responding only.

Further interactions were specific to RSI. First, the RSI $\times f_{0}$ interaction $\left[F(1,11)=11.57, M S_{\mathrm{e}}=3,933.57\right.$, $p=.006]$ revealed that the cost accrued by $f_{0}$ change relative to repetition was apparent at short RSIs (524 vs. $592 \mathrm{msec}$ for $f_{0}$ repeat and change, respectively; Tukey's HSD test; $p<.05$ ), but not at long RSIs (587 vs. $593 \mathrm{msec}$ for $f_{0}$ repeat and change, respectively; Tukey's HSD test; $p>.05)$. Second, the RSI $\times$ task interaction $[F(1,11)=$ 5.68, $\left.M S_{\mathrm{e}}=2,457.45, p=.036\right]$ revealed that the RT slowing associated with long RSIs was greater during the pitch (478 vs. $527 \mathrm{msec}$ for short and long RSIs, respectively) than during the harmonicity task (638 vs. $653 \mathrm{msec}$ for short and long RSIs, respectively). The interaction between task and harmonicity was almost significant $\left[F(1,11)=4.50, M S_{\mathrm{e}}=2,417.59, p=.057\right]$. All other ANOVA terms did not approach statistical significance $[F(1,11)<1.96, p>.190]$.

\section{Discussion}

The data from Experiment 2 replicate a number of findings observed in Experiment 1, using a within-subjects design and different auditory stimuli. Pitch responding was again largely insensitive to changes in both task-relevant $\left(f_{0}\right)$ and task-irrelevant (harmonicity) attributes. In contrast, responding on the basis of harmonicity led to substantial RT costs as a result of stimulus change. First, change in the relevant harmonicity value produced what could be described as a response switching cost, given that participants were required to change responses as a result of harmonicity change. Second, and more importantly, an effect of irrelevant variation was also observed, in that changes in $f_{0}$ were detrimental to harmonicity responding even though these changes were orthogonal to the task-relevant attribute (and therefore, response). The RT costs associated with stimulus change were attenuated as a result of extended RSI, once again indicating that the influence of previously used pathways for processing auditory attributes decays over time. These patterns of data maintain their consistency both with general horse-race models of processing and with specific models of mistuning detection in which periodicity is contingent upon prior frequency information.

\section{EXPERIMENT 3}

The data from Experiments 1 and 2 support the idea that different task requirements for the same acoustic stimuli result in the creation of different processing demands. A third experiment was designed to investigate the neural underpinning of such intertrial repetition effects by examining scalp recording of ERPs. Early discussion of ERP signatures related to acoustic repetition and change fell under the principle of sensory gating (e.g., Fruhstorfer, Soveri, \& 
Järvilehto, 1970). In the simplest case, two identical pure tones are played in succession, and a typical finding is that the ERP amplitude elicited by the second tone is smaller than the amplitude elicited by the first. The idea here is that this reduction in amplitude reflects neural fatigue as a result of quick reactivation of the same neural population, and, as such, it has been used as a low-level assessment tool for comparing clinical samples, such as schizophrenics, with so-called normal samples (e.g., Boutros, Belger, Campbell, D'Souza, \& Krystal, 1998). However, it is possible that at least one contributing factor that influences the direction of the neural effect in response to acoustic repetition is the amount of stimulus uncertainty within the experimental paradigm itself. Under conditions of low stimulus uncertainty in which the (same) stimulus is more likely to repeat than change, neural responses to repetition may become increasingly attenuated as a result of the auditory system adjusting to the overall statistical regularities of the environment. Conversely, and as shown in Dyson, Alain, and $\mathrm{He}$ (2005b), if stimulus uncertainty is high, in that stimulus repetition is the exception rather than the rule, the neural index of repetition can be one of enhancement-for $f_{0}$ repetition, at least. Similar influences of context also apply to later neural indexes of auditory repetition (cf. Haenschel, Vernon, Dwivedi, Gruzelier, \& Baldeweg, 2005; Woods \& Elmasian, 1986).

Previous ERP studies regarding variations in harmonicity have focused on issues such as the location and time course of mistuning processing (Alain et al., 2001; Alain et al., 2002), the interaction between mistuning and location cues in sound segregation (McDonald \& Alain, 2005), and whether the detection of harmonicity can operate in the absence of attention (Alain \& Izenberg, 2003; Dyson \& Alain, 2004; Dyson et al., 2005b). The present experiment extends ideas of harmonic detection by considering the ERP consequences of repeating or changing harmonicity and its relation to pitch change, rather than examining specific values of harmonicity per se (for an examination of the specific effects of stimulus token, see Dyson \& Alain, 2008). One study of direct relevance to Experiment 3 is Dyson et al. (2005b), which found intertrial contingency effects for complex stimuli repeating or changing with respect to $f_{0}$ and harmonicity in ERPs elicited between 30 and $90 \mathrm{msec}$ after sound onset. The results suggested that the auditory system first registers $f_{0}$ as being the same or different, and only if $f_{0}$ repeated was a further distinction made between the repetition or change of harmonicity. Once again, this is consistent with a harmonic template account of mistuning (Lin \& Hartmann, 1998), in that the grouping of harmonics depends on an estimate of $f_{0}$. Therefore, the ability to compare harmonicity information across trials becomes contingent upon the maintenance of the same $f_{0}$, since once the $f_{0}$ of a complex changes, any previous bank of filters (or sieves) set up to evaluate the presence of mistuned energy on the basis of an old $f_{0}$ becomes unusable. This is certainly one generic account of the RT slowing for FDHS trials relative to FSHS trials observed in Experiments 1B and 2B.

Therefore, the ideas put forward by Lin and Hartmann (1998) regarding mistuning detection and the horse-race framework of processing by Mordkoff et al. (1990) once again provide a framework for predicting how ERP modulation in Experiment 3 will be expressed as a result of $f_{0}$ and harmonicity repetition and change. As per Experiments 1 and 2, pitch responding should be faster with $f_{0}$ repetition; this should coincide with shorter ERP latencies, and pitch judgment should be little affected by irrelevant variation harmonicity, resulting in comparable ERPs when harmonicity changes or repeats during the pitch task. On the other hand, a different ERP signature should emerge during the harmonicity task, with variation in task-irrelevant $f_{0}$ causing a delay in ERP latencies. Moreover, if stimulus representations associated with harmonicity processing involve the retrieval of both $f_{0}$ and harmonicity, ERP should be sensitive to both. Also, ERP modulation as a result of harmonicity should be contingent upon the prior repetition of pitch, as per Dyson et al. (2005b). In Experiment 3, we recorded ERPs in conjunction with behavioral measures while participants performed both pitch and harmonicity tasks, and we also collected ERPs elicited by the same stimulus set while participants watched a movie of their choice. This design allowed us to directly compare the mechanisms involved in passively registering as opposed to actively responding to change and repetition in complex sounds. In the present experiment, we focused on the long latency ERP components P2, N2, and P3. P2 is generally thought to be associated with stimulus encoding (Picton \& Fitzgerald, 1983), and as such represents a relatively low-level stage at which the top-down effects of task may reveal their influence. Nevertheless, previous research has shown that P2 amplitude can be modulated as a function of task demands (e.g., Dyson, Alain, \& He, 2005a). In contrast, N2 is thought to be particularly sensitive to the nature of stimulus categorization (cf. Ritter, Simson, Vaughan, \& Macht, 1982), and therefore becomes the most likely candidate to reveal critical ERP modulation when identical stimuli are categorized differently as a function of task demands. Although the exact functional significance of P3 continues to be discussed (see Polich \& Kok, 1995), it is associated with a number of broad-based attentional and memory processes, in addition to response selection (Picton, 1992). Most importantly for the present paradigm, one of the cognitive processes linked to the $\mathrm{P} 3$ is that of contextual stimulus evaluation (Donchin \& Coles, 1988; Hohnsbein, Falkenstein, \& Hoormann, 1995). Therefore, in terms of specific predictions regarding the direction of amplitude effects, we anticipate enhanced ERP amplitude as a result of stimulus repetition under conditions of high stimulus uncertainty, starting around $200 \mathrm{msec}$ after stimulus onset (Dyson \& Alain, 2008; Haenschel et al., 2005) and continuing at least until the time of contextual stimulus evaluation, as represented by the $\mathrm{P} 3$.

\section{Method}

Participants. Informed consent from 12 participants was obtained before the experiment was conducted. Two participants were excluded due to a reported inability to consistently distinguish between tuned and mistuned stimuli and, consequently, 2 additional individuals were run as substitutes. The final sample consisted of 7 females and 5 males, all 12 of whom were right-handed (age range, $21-38$ years; mean $=29.25, S D=5.56$ ). All participants reported normal hearing and received honoraria for their involvement. 
Stimuli and Apparatus. The stimuli were identical to those used in Experiment 2. Stimuli were now routed through a clinical audiometer (GSI 61) and delivered over Eartone 3A insert earphones, although intensity remained at a comfortable level of approximately $75 \mathrm{~dB}$ SPL as measured on the linear scale. Participants completed the experimental session within a sound-attenuated chamber.

Design and Procedure. ERPs were recorded during two active conditions and one passive condition. The two active conditions (pitch and harmonicity) were identical to the passive condition in terms of stimulus set and interstimulus interval. Each trial began with a blank screen for $500 \mathrm{msec}$, followed by the presentation of a 200-msec auditory stimulus, then a time delay averaging $2,100 \mathrm{msec}$, after which a fixation cross flashed in order to indicate the end of the trial. In order to avoid expectancy effects, this delay was jittered $200 \mathrm{msec}$ either side of the average $(1,900,2,100$, or $2,300 \mathrm{msec}){ }^{2}$ Participants were asked to categorize the stimuli as globally "high" or "low" in the active pitch condition (cf. Experiments $1 \mathrm{~A}$ and 2A), and as "tuned/one object" or "mistuned/two objects" in the active harmonicity condition (cf. Experiments 1B and 2B); in the passive condition, they were asked to simply ignore the sounds while watching a muted subtitled movie of their choice. In order to avoid fatigue effects, the passive condition was always completed in the middle of the experimental session (active-passive-active), with the order of the two active conditions counterbalanced across participants. For every condition, participants were exposed to three blocks of stimulus presentation, each lasting approximately $10 \mathrm{~min}$. Each block consisted of the basic set of 16 stimulus pairs ( 4 stimulus contingencies $\times 4$ stimulus tokens) repeated over the various time delays. Orthogonal combinations of trial $n$ and trial $n+1$ time delay were employed, yielding a final block size of 144 stimulus pairs ( 16 stimulus pairs $\times 9$ combinations of delay). Additionally, for each active condition, participants completed a short practice block of 32 trials (16 stimulus pairs presented with a 2,100-msec time lag) before completing the experimental blocks.

Recording of electrophysiological data. Electrical brain activity was digitized continuously (bandpass $0.05-50 \mathrm{~Hz} ; 250-\mathrm{Hz}$ sampling rate) from an array of 64 electrodes using NeuroScan SynAmps and stored for offline analysis. Eye movements were recorded with electrodes placed at the outer canthi and at the superior and inferior orbit. During the data collection, all electrodes were referenced to the midline central electrode (i.e., $\mathrm{Cz}$ ); for data analysis they were rereferenced to the average of all electrodes. Individual epochs included $200 \mathrm{msec}$ of prestimulus activity and $800 \mathrm{msec}$ of poststimulus activity. Epochs contaminated by excessive peak-topeak deflection $( \pm 100 \mu \mathrm{V})$ at the channels not adjacent to the eyes were automatically rejected before averaging. For stimulus presentations at trial $n+1$, ERPs were averaged on the basis of stimulus contingencies (FSHS, FSHD, FDHS, and FDHD) for each of the three experimental conditions (passive, pitch, and harmonicity). In order to achieve equivalent signal-to-noise ratios between active and passive conditions, ERPs were not rejected on the basis of incorrect behavioral responding. In all cases, ERPs were collapsed across the various levels of time delay and averaged separately for each electrode site. For each participant, ocular artifacts (e.g., blinks, saccades, and lateral movements) were corrected by means of ocular source components using the Brain Electrical Source Analysis software (Picton et al., 2000). All ERPs underwent a final low-pass filter to attenuate frequencies above $30 \mathrm{~Hz}$.

\section{Results}

Behavioral data. Errors and outliers in the pitch condition constituted $3.14 \%$ and $2.66 \%$ of total observations; in the harmonicity condition, the respective values were $6.12 \%$ and $2.17 \%$. Table 5 shows the summary statistics for the conditions of interest in Experiment 3. A three-way repeated measures ANOVA involving the factors of task (pitch, harmonicity), $f_{0}$ (repeat, change), and harmonicity (repeat, change) yielded all significant main effects
Table 5

Group Mean Response Times (RTs, in Milliseconds) and Standard Errors (SEs) During the Pitch and Harmonicity Judgment Tasks in Experiment 3

\begin{tabular}{|c|c|c|c|c|}
\hline & RT & $S E$ & $\%$ Error & $\%$ Outlier \\
\hline \multicolumn{5}{|c|}{ Pitch Condition } \\
\hline \multicolumn{5}{|l|}{$f_{0}$ same } \\
\hline Harmonicity same & 411 & 21 & 1.81 & 3.22 \\
\hline Harmonicity different & 444 & 33 & 4.51 & 2.43 \\
\hline \multicolumn{5}{|l|}{$f_{0}$ different } \\
\hline Harmonicity same & 469 & 32 & 3.58 & 1.85 \\
\hline Harmonicity different & 458 & 32 & 2.63 & 3.13 \\
\hline \multicolumn{5}{|c|}{ Harmonicity Condition } \\
\hline \multicolumn{5}{|l|}{$f_{0}$ same } \\
\hline Harmonicity same & 530 & 22 & 2.28 & 3.35 \\
\hline Harmonicity different & 632 & 28 & 9.04 & 1.89 \\
\hline \multicolumn{5}{|l|}{$f_{0}$ different } \\
\hline Harmonicity same & 678 & 30 & 8.68 & 1.32 \\
\hline Harmonicity different & 619 & 27 & 4.47 & 2.13 \\
\hline
\end{tabular}

$\left[F(1,11)=76.43, M S_{\mathrm{e}}=8,993.54, p<.001\right.$ for task; $F(1,11)=44.32, M S_{\mathrm{e}}=1,441.81, p<.001$ for $f_{0}$; and $F(1,11)=6.40, M S_{\mathrm{e}}=988.83, p=.028$ for harmonicity]. The two-way task $\times f_{0}$ and $f_{0} \times$ harmonicity interactions were significant $\left[F(1,11)=20.82, M S_{\mathrm{e}}=289.53, p<\right.$ .001 ; and $F(1,11)=60.47, M S_{\mathrm{e}}=1,052.83, p<.001$, respectively], as was the three-way interaction $[F(1,11)=$ $\left.29.84, M S_{\mathrm{e}}=692.13, p<.001\right]$. Only the task $\times$ harmonicity interaction was not significant $\left[F(1,11)=0.71, M S_{\mathrm{e}}=\right.$ $916.76, p=.417]$. With respect to the significant threeway interaction (see Table 5), Tukey's HSD test showed that in the pitch task, RTs were faster for $f_{0}$ repetition than for change $(p<.05)$. However, in the harmonicity condition, $f_{0}$ change alone (FDHS), harmonicity change alone (FSHD), and both $f_{0}$ and harmonicity change (FDHD) all yielded significantly slower RTs relative to complete stimulus repetition (FSHS; $p<.05$ ). The behavioral data from Experiment 3 replicated a number of findings from Experiments 1 and 2, and support the claim that, for the stimuli used in the present series, responses to pitch can be initiated in the absence of harmonicity, whereas evaluation of the harmonic relations within a complex sound is contingent on $f_{0}$ processing.

ERP data. Figure 1 shows the group average ERPs for cases of complete stimulus repetition (FSHS) recorded during passive, pitch, and harmonicity tasks for all recording sites. In all three listening conditions, ERPs comprised N1, $\mathrm{P} 2$, and $\mathrm{N} 2$ waves peaking at about 110,190 , and $290 \mathrm{msec}$ after stimulus onset, respectively. Both the $\mathrm{N} 1$ and the P2 waves were maximal over the frontocentral scalp sites and inverted in polarity at inferior parietal sites and cerebellar scalp regions, consistent with generators located in the superior temporal plane along the Sylvian fissure. The N2 wave recorded during passive listening was maximal over the central scalp site, but showed a more frontal distribution during the two active listening conditions. In addition to the N1, P2, and N2 waves, the pitch and harmonicity tasks were accompanied by a positive wave peaking at about 390 msec after sound onset. This positive peak, referred to as the P3, appears to be superimposed by a slow wave (SW) that was largest at parietal sites and inverted 
A
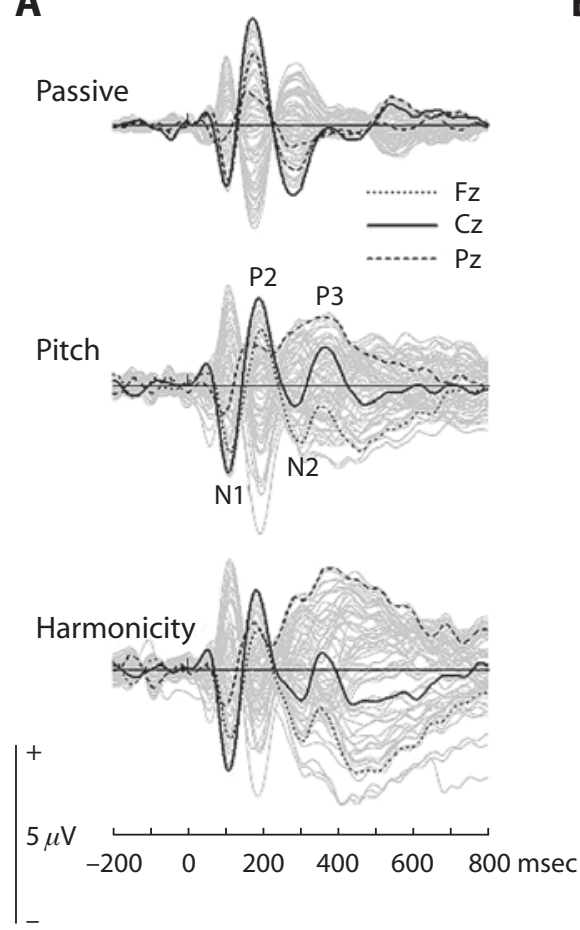

B
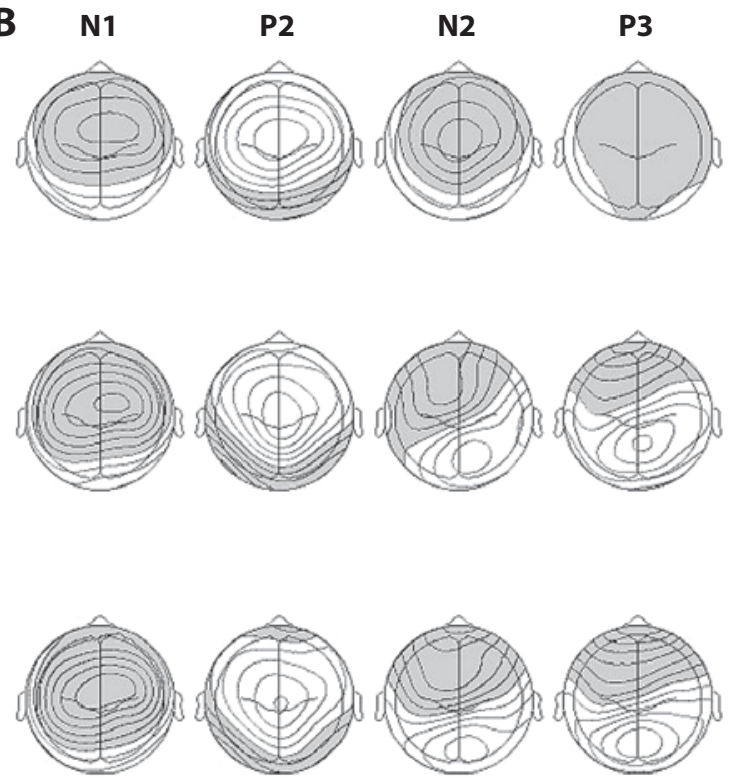

$\mathrm{L} \quad$ top
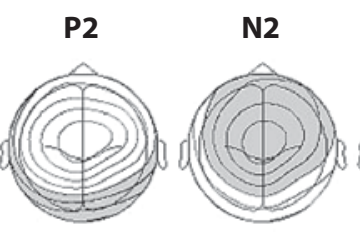

P3

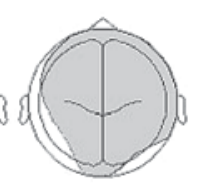

top $\mathrm{R}$

\begin{abstract}
Figure 1. (A) Group mean event-related brain potentials over 65 channels recorded as a result of identical complex sounds repeated over consecutive trials (FSHS). The waveforms represent the two active (pitch and harmonicity) and one passive condition participants completed in Experiment 3. Fz, Cz, and Pz electrodes are shown in black, whereas the other channels are shown in gray. (B) Iso-contour maps are shown for the $\mathrm{N} 1$ (110 msec), P2 (190 msec), N2 (290 msec), and P3 (390 msec) recorded during the passive, pitch, and harmonicity conditions. The contour spacing was set at $.5 \mu \mathrm{V}$. The gray areas indicate negative voltage.
\end{abstract}

in polarity over the frontocentral scalp region. Whereas the SW reversed polarity between anterior and posterior scalp sites, the P3 wave did not, suggesting that it indexed a different process than the SW recorded at frontal and parietal sites.

On the basis of previous research, the effects of intertrial contingencies on peak latency and mean amplitude were explored separately at frontocentral sites for P2, N2, and P3 (e.g., Dyson \& Alain, 2008). Latencies and amplitudes were taken from the mean activity collapsed over six midline frontocentral sites (F1, Fz, F2, FC1, FCz, and FC2) in order to avoid electrode interactions, thereby streamlining the analysis. In all cases, data were analyzed according to three-way repeated measures ANOVAs employing the factors of condition (passive, pitch, harmonicity), $f_{0}$ (same, different), and harmonicity (same, different). Figure 2 shows the group average waveforms recorded from $\mathrm{FCz}$ and $\mathrm{Pz}$ during the three experimental conditions and for the four different types of intertrial contingency.

On the basis of latency estimates from the relevant electrode sites, peak latency and amplitude for P2 were defined as the maximum positivity observed between 150- and 250 -msec poststimulus onset. N2 and P3 were operationalized in similar ways using the epochs $200-300 \mathrm{msec}$ and 300-400 msec, respectively. Mean amplitudes for $\mathrm{P} 2$, N2, and $\mathrm{P} 3$ were quantified according to a $40-\mathrm{msec}$ time window, $20 \mathrm{msec}$ either side of peak latency. This value for mean amplitude was based on the group mean of peak latency over all levels of condition, unless analyses revealed significant differences in peak latency across these factors. In such cases, variations in peak latency for the individual levels of the critical factors were taken into account, and separate mean amplitude time windows were calculated accordingly. Unless otherwise stated, all effects observed in mean amplitude were replicated with peak amplitude and all significant interactions between factors were further explored using Tukey's HSD test $(p<.05)$. Figure 3 provides a graphical representation of peak latency and mean amplitude values for the analyses of P2, N2, and P3 over frontocentral sites, and Table 6 provides the ANOVA summaries for all ERP components.

P2. As can be seen in Figure 2 and the top left panel of Figure 3, the peak of the $\mathrm{P} 2$ wave occurred earlier when the value of $f_{0}$ was maintained across trials $(194 \mathrm{msec})$, as opposed to when it changed $(210 \mathrm{msec} ; p<.001)$. Given the absence of a $f_{0} \times$ condition interaction, this kind of sensitivity to the repetition or change of $f_{0}$ appeared to be a common feature within all conditions, and consequently may reflect a mandatory aspect of acoustic processing. With respect to P2 mean amplitude, there were significant main effects of condition ( $p=.004)$ and harmonicity $(p=.014)$, as well as a two-way condition $\times$ harmonicity interaction $(p=.039)$. This interaction revealed larger $\mathrm{P} 2$ amplitude when harmonicity repeated rather than changed 
Frontal (FCz)
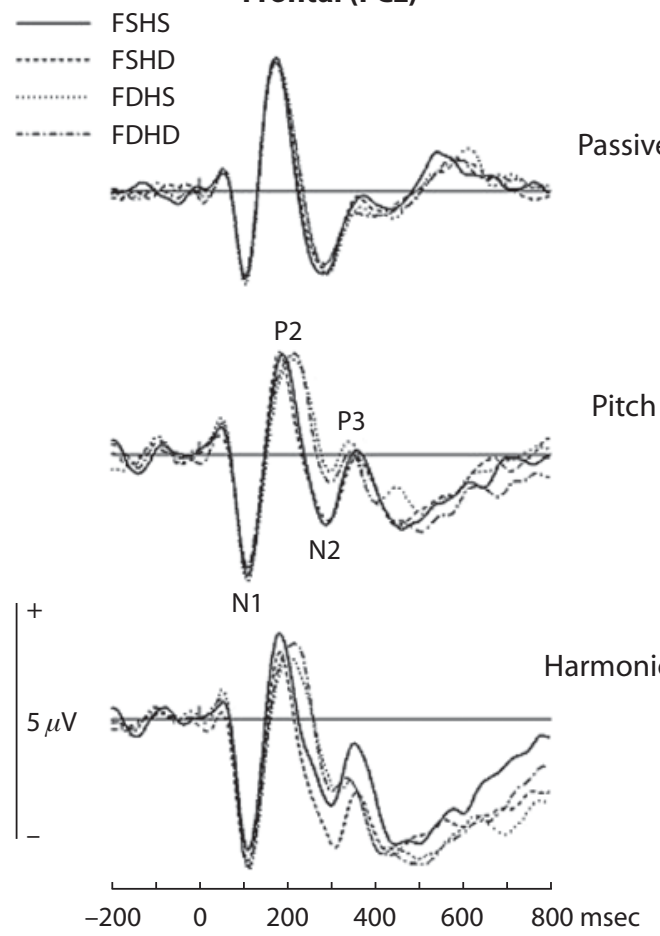

Parietal (Pz)

Passive

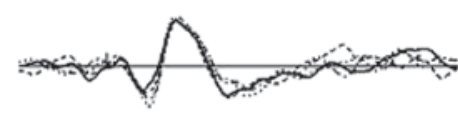

Figure 2. Group mean event-related brain potentials recorded from FCz and Pz during Experiment 3 over three conditions: passive, pitch, and harmonicity, respectively. The waveforms vary as a function of intertrial contingency, in which both $f_{0}$ and harmonicity could have the same or different values across trials $n$ and $n+1$. FS, frequency same; FD, frequency different; HS, harmonicity same; HD, harmonicity different.

Table 6

Summary of Separate ANOVAs for Peak Latency and

Mean Amplitude for P2, N2, and P3 in Experiment 3 at

Frontocentral Sites (F1, Fz, F2, FC1, FCz, and FC2)

\begin{tabular}{|c|c|c|c|c|c|c|}
\hline \multirow{2}{*}{$\begin{array}{l}\text { Component } \\
\text { Factor }(d f)\end{array}$} & \multicolumn{3}{|c|}{ Peak Latency } & \multicolumn{3}{|c|}{ Mean Amplitude } \\
\hline & $F$ & $M S_{\mathrm{e}}$ & $p$ & $F$ & $M S_{\mathrm{e}}$ & $p$ \\
\hline \multicolumn{7}{|l|}{$\mathrm{P} 2$} \\
\hline $\mathrm{C}(2,22)$ & 0.11 & 394.41 & .892 & 7.36 & 2.44 & .004 \\
\hline $\mathrm{F}(1,11)$ & 21.75 & 407.20 & $<.001$ & 0.01 & 0.84 & .926 \\
\hline $\mathrm{H}(1,11)$ & 0.63 & 118.93 & .444 & 8.54 & 0.18 & .014 \\
\hline $\mathrm{C} \times \mathrm{F}(2,22)$ & 1.41 & 174.29 & .264 & 0.92 & 0.34 & .413 \\
\hline $\mathrm{C} \times \mathrm{H}(2,22)$ & 1.27 & 114.61 & .300 & 3.79 & 0.13 & .039 \\
\hline $\mathrm{F} \times \mathrm{H}(1,11)$ & 0.13 & 162.97 & .722 & 4.26 & 0.28 & .063 \\
\hline $\mathrm{C} \times \mathrm{F} \times \mathrm{H}(2,22)$ & 1.29 & 115.88 & .296 & 1.82 & 0.18 & .185 \\
\hline \multicolumn{7}{|l|}{ N2 } \\
\hline $\mathrm{C}(2,22)$ & 0.55 & 905.05 & .584 & 33.06 & 1.06 & $<.001$ \\
\hline $\mathrm{F}(1,11)$ & 2.90 & 335.09 & .116 & 11.59 & 0.74 & $<.001$ \\
\hline $\mathrm{H}(1,11)$ & 2.47 & 113.51 & .144 & 6.79 & 0.20 & .024 \\
\hline $\mathrm{C} \times \mathrm{F}(2,22)$ & 0.40 & 152.57 & .677 & 2.68 & 0.37 & .091 \\
\hline $\mathrm{C} \times \mathrm{H}(2,22)$ & 2.96 & 166.57 & .073 & 0.92 & 0.40 & .414 \\
\hline $\mathrm{F} \times \mathrm{H}(1,11)$ & 0.06 & 190.90 & .812 & 2.49 & 0.16 & .142 \\
\hline $\mathrm{C} \times \mathrm{F} \times \mathrm{H}(2,22)$ & 0.80 & 237.42 & .462 & 4.15 & 0.26 & .029 \\
\hline \multicolumn{7}{|l|}{ P3 } \\
\hline $\mathrm{C}(2,22)$ & 0.55 & 998.69 & .947 & 23.87 & 1.40 & $<.001$ \\
\hline $\mathrm{F}(1,11)$ & 8.06 & 888.87 & .016 & 0.42 & 0.66 & .528 \\
\hline $\mathrm{H}(1,11)$ & 0.67 & 223.57 & .429 & 8.60 & 0.21 & .014 \\
\hline $\mathrm{C} \times \mathrm{F}(2,22)$ & 0.09 & 351.63 & .915 & 0.21 & 0.43 & .814 \\
\hline $\mathrm{C} \times \mathrm{H}(2,22)$ & 0.14 & 334.75 & .869 & 1.06 & 0.29 & .363 \\
\hline $\mathrm{F} \times \mathrm{H}(1,11)$ & 0.24 & 155.62 & .637 & 1.65 & 0.33 & .225 \\
\hline $\mathrm{C} \times \mathrm{F} \times \mathrm{H}(2,22)$ & 0.03 & 374.54 & .971 & 5.71 & 0.30 & .010 \\
\hline
\end{tabular}

Note-C, condition; $\mathrm{F}, f_{0} ; \mathrm{H}$, harmonicity. 
Peak Latency
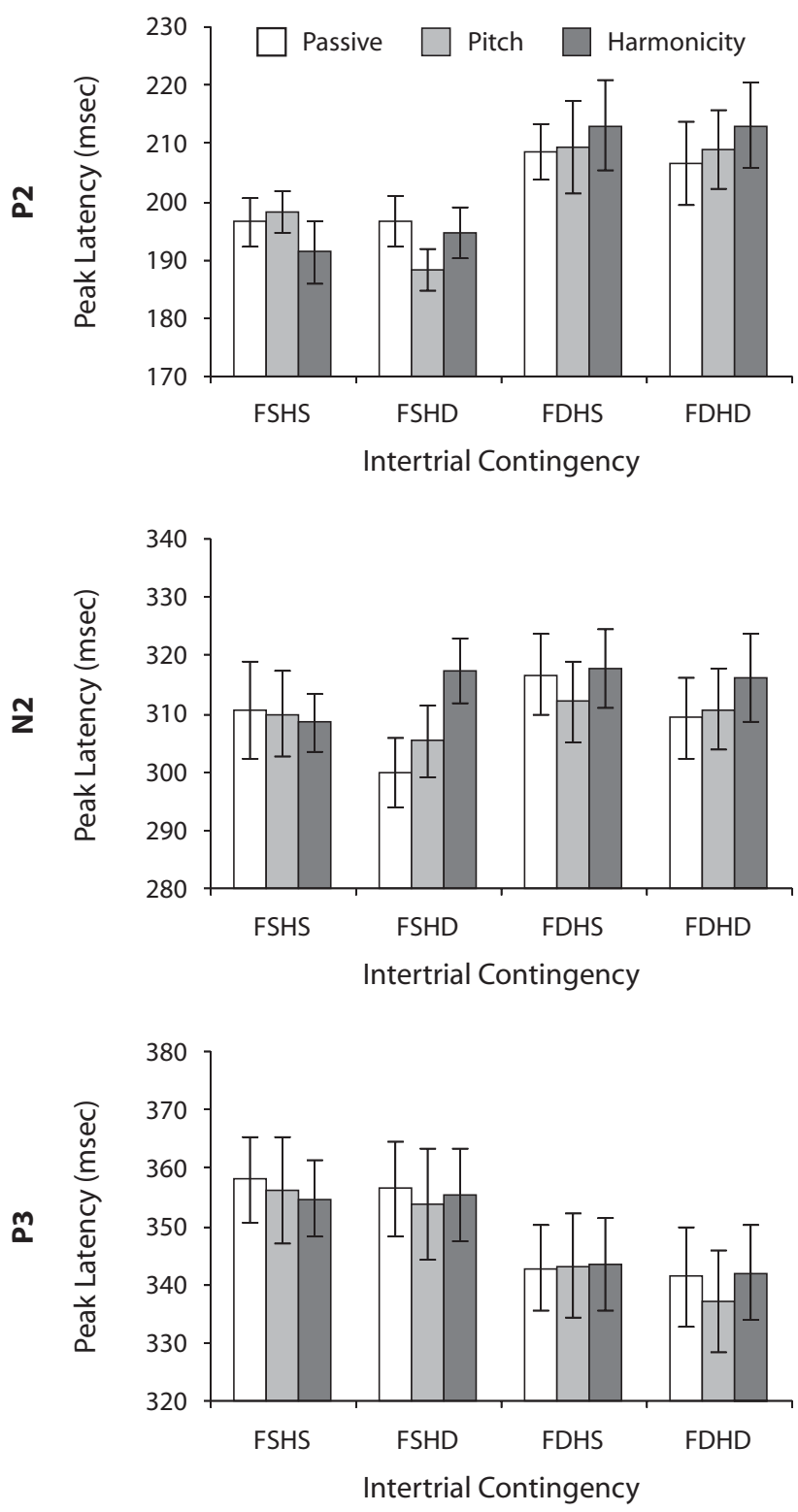

Mean Amplitude
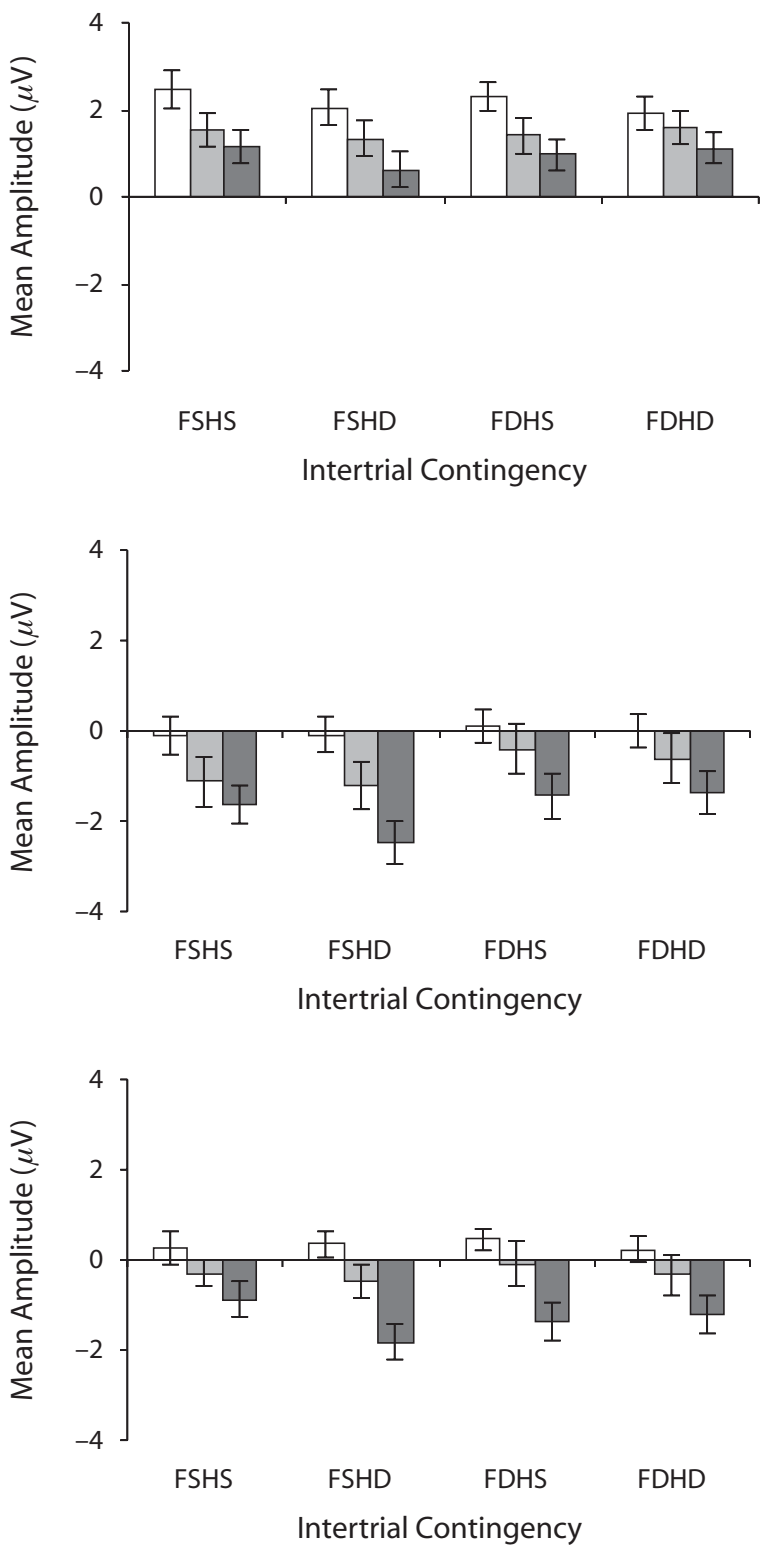

Figure 3. Group peak latency and mean amplitude for P2, N2, and P3 generated in Experiment 3 over frontocentral sites. ERP components are shown over three experimental conditions (passive, pitch, harmonicity), each with the same four intertrial contingencies between trials $\boldsymbol{n}$ and $\boldsymbol{n}+1$. Intertrial contingency represents the orthogonal combination of two levels of $\boldsymbol{f}_{0}$ (same, different) and two levels of harmonicity (same, different). FS, frequency same; FD, frequency different; HS, harmonicity same; HD, harmonicity different. Error bars represent standard errors of the means.

in the passive condition only. However, this effect must be interpreted with respect to a two-way interaction between $f_{0}$ and harmonicity, which approached statistical significance $(p=.063)$. Here, $\mathrm{P} 2$ modulation as a result of the repetition or change of harmonicity was greater for cases of $f_{0}$ repetition. In sum, $\mathrm{P} 2$ amplitude was more pronounced for cases of complete stimulus repetition (FSHS) in the passive condition. Although small, the effect is consistent with the proposal that registering invariance within an acoustic object over time can occur without top-down control (Dyson et al., 2005b).
N2. No main effects or interactions were revealed for N2 peak latency. Mean amplitude produced main effects for condition $(p<.001), f_{0}(p<.001)$, harmonicity $(p=$ $.024)$, and a three-way interaction between all factors ( $p=$ .029). Decomposition of the three-way interaction showed larger N2 amplitude for the harmonicity condition, and a larger response in the harmonicity condition when $f_{0}$ was maintained but harmonicity changed (FSHD) across trial, relative to the three other types of intertrial contingency. A secondary aspect of the data was more clearly revealed by peak amplitude measurements - namely, a two-way interac- 
tion between condition and $f_{0}(p=.040)$. Here, larger N2 responses were generated for cases of $f_{0}$ repetition as opposed to $f_{0}$ change (cf. Haenschel et al., 2005), but only during the pitch condition (see Figure 2). This effect was only partially replicated by the three-way interaction in mean amplitude, revealing larger N2 amplitude in the pitch condition for the comparison between FSHD and FDHS trials only.

The results of N2 amplitude are particularly important, since this ERP component shows differential modulation according to task demands (cf. Ritter et al., 1982), with N2 activity in both the pitch and the harmonicity condition providing consistency with the behavioral data. First, in the pitch condition, there was clear separation in N2 amplitude on the basis of $f_{0}$ repetition or change. This is consistent with the observation that during pitch processing, participants seem to reliably retain only pitch information across trials. Second, in the harmonicity condition, N2 modulation was sensitive to harmonicity but only when the $f_{0}$ was maintained. This is consistent with the idea that during harmonicity processing, any comparison of previous and current values of (mis)tuning relies on the prior registration of $f_{0}$.

P3. At frontocentral sites, the P3 peak latency was delayed following $f_{0}$ repetition ( $356 \mathrm{msec}$ ) relative to change (342 msec; $p=.033$ ). The condition-independent nature of this effect once again suggests an automatic neural response to $f_{0}$ repetition, in a manner similar to that reported for P2 peak latency. With P3 mean amplitude, the main effects of condition $(p<.001)$ and harmonicity repetition $(p=.014)$ were statistically significant, in addition to a three-way condition $\times f_{0} \times$ harmonicity interaction $(p=.010)$. Further decomposition of the three-way interaction showed that P3 amplitude was larger in the harmonicity condition relative to the passive and pitch conditions. This observation replicates a common finding in the ERP literature: that P3 amplitude reflects an RT main effect of condition (Patel \& Azzam, 2005), with larger (and often later) P3 apparent in the condition with the slowest RT. For the harmonicity condition, cases of $f_{0}$ and harmonicity repetition (FSHS) generated larger $\mathrm{P} 3$ amplitude relative to instances in which $f_{0}$ repeated but harmonicity changed (FSHD; see Figure 2). ${ }^{3}$ Like N2 amplitude, P3 amplitude shows ERP modulation in the harmonicity condition in which the registration of (mis)tuning appears to be contingent upon the repetition of $f_{0}$.

\section{Discussion}

The behavioral data from Experiment 3 replicated the main findings of Experiments 1 and 2 in that only estimates of $f_{0}$ appeared to be retained for complex sounds submitted to pitch categorization, whereas representation of both $f_{0}$ and harmonicity appeared to influence listeners' ability to judge harmonicity. We expected to see these patterns of performance reflected in ERP, and four main findings are relevant here. First, significant differences in P2 and P3 latency were observed at frontocentral sites, according to whether $f_{0}$ was maintained or changed across trials. Specifically, earlier P2 and later P3 latencies were observed for an acoustic stimulus that repeated with respect to $f_{0}$. Although the functional significance of this finding remains an issue for future studies, it seems reasonable that the maintenance of pitch across trials should allow for quicker and extended access to central auditory processing as represented by the temporal window between P2 and P3. Moreover, this neural response is also likely to be an automatic consequence of $f_{0}$ repetition, given that latency differences in $\mathrm{P} 2$ and $\mathrm{P} 3$ were observed across all experimental conditions, including a passive condition in which participants were not required to actively attend to the stimuli. A question remains as to whether latency shifts best index modulation of a single ERP component, or the activation of an additional ERP component, which overlaps the deflection of interest. Regardless of the putative mechanism behind latency shifts, on the basis of these effects we argue that, in the present experiment, $f_{0}$ is compared across consecutive trials in a stimulus-driven manner undisturbed by task.

Second, N2 showed differential sensitivity to intertrial contingency as a function of the type of active processing currently being undertaken. During the pitch condition, N2 amplitude distinguished between cases of $f_{0}$ repetition and change and did not reliably dissociate between cases of harmonicity change or repetition for either value of $f_{0}$. During harmonicity processing, however, N2 amplitude showed sensitivity to harmonicity that was contingent upon $f_{0}$ repetition. In this respect, $\mathrm{N} 2$ appears to be a strong candidate for indexing the active comparison between the incoming stimulus and the previous sound as a function of task demands (Ritter et al., 1982).

Third, complete stimulus repetition (FSHS trials) was indexed by larger P3 amplitude at frontocentral sites in the harmonicity condition only. This supports the notion that both pitch and harmonicity attributes are encoded, stored, and compared during harmonicity responding, a contrast to pitch judgments, in which only $f_{0}$ appears to play an important role in guiding participants' responses. Specifically, the increase in $\mathrm{P} 3$ amplitude for cases in which the experimental stimulus does not change is consistent with the idea that P3 may reflect context updating (Donchin \& Coles, 1988; Hohnsbein et al., 1995). That is, when a stimulus is liable to change $75 \%$ of the time across trial either on one or both attributes, as in the present designs, complete stimulus repetition occurring on $25 \%$ of trials represents the greatest violation to an experimental environment defined by change. Larger P3 amplitude as a result of stimulus maintenance may also act as a neural marker for the bypassing of perceptual processing as a result of complete stimulus repetition (Pashler \& Baylis, 1991; Rabbitt, 1992). Although alternative accounts of $\mathrm{P} 3$ repetition effects are available (e.g., Sommer, Matt, \& Leuthold, 1990, p. 914), these approaches do not appear to predict the specific stimulus-based effects observed in Experiment 3 and the way in which harmonicity modulation is contingent upon $f_{0}$ repetition when responding to frequency periodicity only.

Fourth, it is important to note that stimulus maintenance also resulted in smaller SW activity observed after P3 for FSHS trials in the harmonicity condition (see Figure 2). In the present context, modulation in SW activity at trial $n+1$ is particularly interesting, since it hints at the relentless nature of repetition effects in that trial $n+1$ is going to have an effect on trial $n+2$. It is worth stressing again that SW modulation occurs after participants have responded to 
the second trial in any given stimulus pair. Consequently, SW modulation goes beyond the remit of the present data set and demonstrates that contextual effects observed in the present experimental series are not limited to "firstorder" repetitions (i.e., trial $n$ to trial $n+1$ ), but may extend to "higher-order" repetitions (i.e., trial $n$ to trial $n+1$ to trial $n+2$, etc.; Kirby, 1980; Soetens, Boer, \& Hueting, 1985; Sommer, Leuthold, \& Soetens, 1999). Processing facilitation observed as a result of complete stimulus repetition over a number of iterations makes sense from an ecological perspective, since perceptual invariance over time would be a strong indicator that a temporally stable object is present in the environment.

Taken as a whole, it is useful to contrast the present data set with the observations of Dyson et al. (2005b), who examined the repetition and change of complex sounds within primary and secondary auditory cortical areas using middle latency auditory-evoked responses. Their data suggest that the auditory system soon arrives at a veridical representation of the acoustic environment, in which all salient aspects of a sound are registered and compared on a momentto-moment basis in the absence of attention. Whereas the present data suggest that pitch repetition or change is represented independently of task demands (cf. latency modulation of P2), at higher cortical levels, such as those involved in the generation of later ERP components such as N2 and P3 (Picton, 1992; Picton \& Fitzgerald, 1983), it seems as though stimulus representations show sensitivity to only those attributes critical for the completion of the current task. We may synthesize these two approaches and argue that, in the interests of an efficient processing system, we require an early series of computations to register all aspects of our sensory environments for regularities and a later set of processes to re-form this veridical representation. In this way, the specific attributes we need to complete the task at hand would become the focus of computation.

\section{GENERAL DISCUSSION}

\section{Pitch and Harmonicity Processing}

In the present experimental series, $f_{0}$ processing was shown to be largely independent of harmonicity registration, but the detection of tuning or mistuning appeared to be contingent upon prior $f_{0}$ analysis. In this respect, the data support the coding of $f_{0}$ into the global representation of an acoustic scene unfolding over time, as in Darwin et al. (1995), but also extend this observation beyond streaming paradigms to procedures involving carryover effects produced by equiprobable intertrial contingencies (Olivers \& Humphreys, 2003). However, the processing of harmonicity did not appear to be a mandatory exercise at higher cortical levels, as evidenced by the marginal influence of (mis)tuning on pitch judgments, with respect to both behavioral and ERP measures.

The nature of this interaction is also consistent with current models of harmonic detection (Duifhuis et al., 1982; Lin \& Hartmann, 1998; Scheffers, 1983). The harmonic template models argue that decisions regarding harmonic relations within a complex sound are based on (1) an estimate of $f_{0}$ and (2) subsequent sensitivity to higher frequencies that are integer multiples of that $f_{0}$. Therefore, neurons that are sensitive to the dominant periodic acoustic signal (i.e., $f_{0}$ ) may act as a filter bank that allows harmonically related partials to fall through the template, leaving unharmonically related portions of acoustic energy to form distinct perceptual objects (for a review, see Alain, 2007). Therefore, although the standard auditory phenomenology of mistuning is one of perceptual "pop-out," our results are consistent with current theories of harmonicity and show that mistuning cannot be detected without an estimation of $f_{0}$.

\section{Acoustic Discriminability}

Taking a term derived from the visual literature, the specific interaction between pitch and harmonicity observed here might be more broadly characterized as an example of an asymmetrical separable relationship (Garner, 1976). The style of interaction between a pair of asymmetrical separable acoustic attributes is described by Mordkoff and Yantis (1991; see also Mordkoff et al., 1990) in terms of the typical failure of selective attention when one is attempting to process the slower dimension and ignore the faster dimension, and the typical success of selective attention when one is attempting to process the faster dimension and ignore the slower dimension (see also Melara \& Mounts, 1993). Our data are consistent with such a model, since pitch judgments tended to be faster than harmonicity judgments; pitch had a systematic slowing effect on harmonicity responding, whereas harmonicity did not have a systematic slowing effect on pitch responding.

However, there remains the possibility that the current interaction between $f_{0}$ and harmonicity may actually be a special case of an asymmetrical separable relationship whereby one stimulus attribute (i.e., harmonicity) is dependent on the output of a second attribute (i.e., $f_{0}$ ): A strict interpretation of the harmonic template model was that harmonicity judgment could never be as fast as $f_{0}$ judgments. Therefore, it is necessary to address to what extent the nature of this asymmetrical separable relationship is simply a by-product of different speeds of processing. It could be argued that by increasing the salience of the tuned/mistuned distinction and reducing $f_{0}$ difference, the detection of harmonicity could become faster than pitch, leading to the reversal of the asymmetric separable relationship, in that harmonicity would reliably interfere with pitch judgments but not vice versa. There are a number of reasons why this may not be so. First, the current level of mistuning $(16 \%)$ of a single low harmonic partial leads to a clear and unambiguous percept of two concurrent auditory objects, as supported by previous behavioral data (Alain et al., 2001; Alain et al., 2002). Second, differences in $f_{0}$ were decreased from $200 \mathrm{~Hz}$ in Experiment 1 to $132 \mathrm{~Hz}$ in Experiments 2 and 3, with little discernible effect on the magnitude of the effect (see Tables 1-5). Third, further reductions in $f_{0}$ differences between complex sounds are likely to create a confound by encouraging participants to focus on specific frequency regions: As $f_{0} \mathrm{~s}$ become closer, so too will the frequencies defining the (mis)tuned partial in each case, if these are defined within the same partial. In this respect, we return to the potential problem of perceptual "pop-out" of particular harmonic regions, whether 
or not the region contains a tuned or mistuned element (see discussion of Experiment 1B). Fourth, if differences in speeds of processing between $f_{0}$ and harmonicity were critical in determining the amount of interference that $f_{0}$ change had on harmonicity, this should be reflected in a positive correlation between the magnitude of RT differences associated with harmonicity processing relative to pitch processing and the RT cost associated with changing $f_{0}$ in the harmonicity condition. However, the data from Experiments 2 and 3, in which individuals completed both pitch and harmonicity conditions, reveal no such correlation $[r(24)=-.009, p=.966]$. Therefore, the magnitude of $f_{0}$ interference on harmonicity processing did not appear to be contingent upon differences in speeds of processing between $f_{0}$ and harmonicity. The idea of harmonicity being contingent upon $f_{0}$ is further supported by the observation that, in contrast to other combinations of auditory dimensions such as location and pitch (Dyson \& Quinlan, 2004), RTs for complete stimulus repetition (FSHS) were different, according to whether participants completed the pitch or the harmonicity condition (see Table 5).

\section{A Special Role for Frequency?}

In contrast to standard horse-race accounts of stimulus processing, previous data have also shown that slowing the processing speed of pitch does not necessarily stop it from interfering with other acoustic attributes. Mondor, Zattore, and Terrio (1998) report a case in which left-right location and high-low pitch responding were compared using attribute variation similar to that in the present paradigm. Although location was processed faster than pitch on average, irrelevant changes in pitch across consecutive trials still exerted a detrimental effect on RTs during location processing (see also Experiment 3 in Dyson \& Quinlan, 2004). These kinds of observations support the idea of a special role for frequency within audition (see also Kubovy, 1981; Kubovy \& Van Valkenburg, 2001; Purves et al., 1997; Woods, Alain, Diaz, Rhodes, \& Ogawa, 2001), in that participants are seemingly unable to ignore the processing of frequency during acoustic stimulation. Consistent with this data, the ERPs from Experiment 3 provide support for this specific role, in the conditionindependent elongation of the processing period between P2 and $\mathrm{P} 3$ for cases of $f_{0}$ repetition (see Figure 3 ).

\section{Conclusion}

Findings from this experimental series suggest that the acoustic attributes of pitch and harmonicity share a relationship in which pitch information is available independently of harmonicity but harmonic processing is contingent upon the prior registration of pitch. This was revealed by both behavioral and ERP measures. Our results show that the auditory system detects the presence of tuning or mistuning within complex sounds on the basis of fundamental frequency, and the same results support notions of the special role of frequency within the acoustic system at large. The data also demonstrate the utility of repetition effects by revealing the mechanisms involved in a topdown processing system that shapes the bottom-up acoustic input into representations that are merely sufficient for the task at hand.

\section{AUTHOR NOTE}

Part of this article was presented at the 4th Auditory Perception, Cognition, and Action Meeting, November 10, 2005, in Toronto. The authors thank Kelly McDonald and Yu He for their assistance in the development of this work, and a number of anonymous reviewers for their comments relating to earlier drafts of the manuscript. This research was funded by grants from the Canadian Institute for Health Research, the Natural Sciences and Engineering Research Council of Canada, the Premier Research Excellence Award from the Ontario Government, Deafness Research UK, and the Royal National Institute for the Deaf. Correspondence concerning this article should be addressed to B. J. Dyson, Department of Psychology, Ryerson University, 350 Victoria Street, Toronto, ON, M5B 2K3 Canada (e-mail: ben.dyson@psych.ryerson.ca).

\section{REFERENCES}

AlaIN, C. (2007). Breaking the wave: Effects of attention and learning on concurrent sound perception. Hearing Research, 229, 225-236.

Alain, C., Arnott, S. R., \& Picton, T. W. (2001). Bottom-up and topdown influences on auditory scene analysis: Evidence from eventrelated brain potentials. Journal of Experimental Psychology: Human Perception \& Performance, 27, 1072-1089.

AlAin, C., \& IzENBERG, A. (2003). Effects of attentional load on auditory scene analysis. Journal of Cognitive Neuroscience, 15, 1063-1073.

Alain, C., Schuler, B. M., \& McDonald, K. L. (2002). Neural activity associated with distinguishing concurrent auditory objects. Journal of the Acoustical Society of America, 111, 990-995.

BERTELSON, P. (1961). Sequential redundancy and speed in a serial twochoice responding task. Quarterly Journal of Experimental Psychology, 13, 90-102.

Boutros, N. N., Belger, A., Campbell, D., D'Souza, C., \& KrysTAL, J. (1998). Comparison of four components of sensory gating in schizophrenia and normal subjects: A preliminary report. Psychiatry Research, 88, 119-130.

Bregman, A. S. (1990). Auditory scene analysis. Cambridge, MA: MIT Press.

Cusack, R., Deeks, J., Aikman, G., \& Carlyon, R. P. (2004). Effects of location, frequency region, and time course of selective attention on auditory scene analysis. Journal of Experimental Psychology: Human Perception \& Performance, 30, 643-656.

Darwin, C. J., \& Ciocca, V. (1992). Grouping in pitch perception: Effects of onset asynchrony and ear of presentation of a mistuned component. Journal of the Acoustical Society of America, 91, 3381-3390.

Darwin, C. J., Ciocca, V., \& Sandell, G. J. (1994). Effects of frequency and amplitude modulation on the pitch of a complex tone with a mistuned harmonic. Journal of the Acoustical Society of America, 95, 2631-2636.

Darwin, C. J., Hukin, R. W., \& Al-Khatib, B. Y. (1995). Grouping in pitch perception: Evidence for sequential constraints. Journal of the Acoustical Society of America, 98, 880-885.

Donchin, E., \& Coles, M. G. H. (1988). Precommentary: Is the P300 component a manifestation of context updating? Behavioral \& Brain Sciences, 11, 355-435.

Duifhuis, H., Willems, L. F., \& Sluyter, R. J. (1982). Measurement of pitch in speech: An implementation of Goldstein's theory of pitch perception. Journal of the Acoustical Society of America, 71, 1568-1580.

Dyson, B. J. (in press). Perceptual organisation. In C. Plack (Ed.), The Oxford handbook of auditory science: Auditory perception. Oxford: Oxford University Press.

Dyson, B. J., \& ALAIN, C. (2004). Representation of concurrent auditory objects in primary auditory cortex. Journal of the Acoustical Society of America, 115, 280-289.

Dyson, B. J., \& AlaIn, C. (2008). Is a change as good with a rest? Task dependent effects of inter-trial contingency on concurrent sound segregation. Brain Research, 1189, 135-144.

Dyson, B. J., Alain, C., \& He, Y. (2005a). Effect of visual attentional load on auditory scene analysis. Cognitive, Affective, \& Behavioral Neuroscience, 5, 319-338.

Dyson, B. J., Alain, C., \& He, Y. (2005b). I've heard it all before: Perceptual invariance represented by early cortical auditory evoked responses. Cognitive Brain Research, 23, 457-460. 
Dyson, B. J., \& Quinlan, P. T. (2004). Stimulus processing constraints in audition. Journal of Experimental Psychology: Human Perception \& Performance, 30, 1117-1131

Fruhstorfer, H., Soveri, P., \& Järvilehto, T. (1970). Short-term habituation of the auditory evoked response in man. Electroencephalography \& Clinical Neurophysiology, 28, 153-161.

GARNER, W. R. (1976). Interaction of stimulus dimensions in concept and choice processes. Cognitive Psychology, 8, 98-123.

Haenschel, C., Vernon, D. J., Dwivedi, P., Gruzelier, J. H., \& BALDEWEG, T. (2005). Event-related brain potential correlates of human auditory sensory memory-trace formation. Journal of Neuroscience, 25, 10494-10501.

Hartmann, W. M. (1988). Pitch perception and the segregation and the integration of auditory entities. In G. M. Edelman, W. E. Gall, \& W. M. Cowan (Eds.), Auditory function: Neurobiological bases of hearing (pp. 623-645). New York: Wiley.

Hohnsbein, J., Falkenstein, M., \& Hoormann, J. (1995). Effects of attention and time-pressure on $\mathrm{P} 300$ subcomponents and implications for mental workload research. Biological Psychology, 40, 73-81.

HyMAN, R. (1953). Stimulus information as a determinant of reaction time. Journal of Experimental Psychology, 45, 188-196.

JoLiCGEUR, P. (1999). Concurrent response-selection demands modulate the attentional blink. Journal of Experimental Psychology: Human Perception \& Performance, 25, 1097-1113.

KIRBY, N. (1980). Sequential effects in choice reaction time. In A. T. Welford (Ed.), Reaction times (pp. 129-172). London: Academic Press.

Kornblum, S. (1973). Sequential effects in choice reaction time: A tutorial review. In S. Kornblum (Ed.), Attention and performance IV (pp. 259-288). New York: Academic Press.

KrUger, L. E., \& Shapiro, R. G. (1981). Intertrial effects of samedifferent judgements. Quarterly Journal of Experimental Psychology, 33A, 241-265.

Kubovy, M. (1981). Concurrent-pitch segregation and that theory of indispensable attributes. In M. Kubovy \& J. R. Pomerantz (Eds.), Perceptual organization (pp. 55-98). Hillsdale, NJ: Erlbaum.

Kubovy, M., \& VAN VALKenburG, D. (2001). Auditory and visual objects. Cognition, 80, 97-126.

LiN, J.-Y., \& HARTMANN, W. M. (1998). The pitch of a mistuned harmonic: Evidence for a template model. Journal of the Acoustical Society of America, 103, 2608-2617.

MCDonald, K. L., \& Alain, C. (2005). Contribution of harmonicity and location to auditory object formation in free field: Evidence from event-related brain potentials. Journal of the Acoustical Society of America, 118, 1593-1604.

Melara, R. D., \& MounTs, J. R. W. (1993). Selective attention to Stroop dimensions: Effects of baseline discriminability, response mode, and practice. Memory \& Cognition, 21, 627-645.

Mondor, T. A., Zatorre, R. J., \& Terrio, N. A. (1998). Constraints on the selection of auditory information. Journal of Experimental Psychology: Human Perception \& Performance, 24, 66-79.

Moore, B. C. J., Glasberg, B. R., \& Peters, R. W. (1986). Thresholds for hearing mistuned partials as separate tones in harmonic complexes. Journal of the Acoustical Society of America, 80, 479-483.

MordKofF, J. T., \& YANTIS, S. (1991). An interactive race model of divided attention. Journal of Experimental Psychology: Human Perception \& Performance, 17, 520-538.

Mordkoff, J. T., Yantis, S., \& Egeth, H. E. (1990). Detecting conjunctions of color and form in parallel. Perception \& Psychophysics, 48, 157-168.

Olivers, C. N. L., \& Humphreys, G. W. (2003). Attentional guidance by salient feature singletons depends on intertrial contingencies. Journal of Experimental Psychology: Human Perception \& Performance, 29, 650-657.

PAshler, H., \& BAYlis, G. (1991). Procedural learning: 2. Intertrial repetition effects in speeded-choice judgments. Journal of Experimental Psychology: Learning, Memory, \& Cognition, 17, 33-48.

Patel, S. H., \& Azzam, P. N. (2005). Characterization of N200 and P300: Selected studies of the event-related potential. International Journal of Medical Sciences, 2, 147-154.

Picton, T. W. (1992). The P300 wave of the human event-related potential. Journal of Clinical Neurophysiology, 9, 456-479.

Picton, T. W., \& Fitzgerald, P. G. (1983). A general description of the human auditory evoked potentials. In E. J. Moore (Ed.), Bases of auditory brain-stem evoked responses (pp. 141-156). New York: Grune \& Stratton.

Picton, T. W., van Roon, P., Armilio, M. L., Berg, P., Ille, N., \& Scherg, M. (2000). The correction of ocular artifacts: A topographic perspective. Clinical Neurophysiology, 111, 53-65.

Polich, J., \& KoK, A. (1995). Cognitive and biological determinants of P300: An integrative review. Biological Psychology, 41, 103-146.

Purves, D., Augustine, G. J., Fitzpatrick, D., Katz, L. C., LaMantia, A. S., \& McNamara, J. O. (EdS.) (1997). Neuroscience. Sunderland, MA: Sinauer.

Quinlan, P. T. (1999). Sequential effects in auditory choice reaction time tasks. Psychonomic Bulletin \& Review, 6, 297-303.

RabiItT, P. (1992). Many happy repetitions: A celebration of the "Berelson Repetition Effect," 1961-1991. In J. Alegria, D. Holender, J. Junca de Morais, \& M. Radeau (Eds.), Analytic approaches to human cognition (pp. 313-330). Amsterdam: Elsevier.

Reinke, K. S., He, Y., WANG, C., \& Alain, C. (2003). Perceptual learning modulates sensory evoked response during vowel segregation. Cognitive Brain Research, 17, 781-791.

Ritter, W., Simson, R., Vaughan, H. G., JR., \& Macht, M. (1982). Manipulation of event-related potential manifestations of information processing stages. Science, 218, 909-911.

Roberts, B., \& Holmes, S. D. (2006). Grouping and the pitch of a mistuned fundamental component: Effects of applying simultaneous multiple mistunings to the other harmonics. Hearing Research, 222, $79-88$.

SCHEFFERS, M. T. M. (1983). Simulation of auditory analysis of pitch: An elaboration on the DWS pitch meter. Journal of the Acoustical Society of America, 74, 1716-1725.

Soetens, E., Boer, L. C., \& Hueting, J. E. (1985). Expectancy or automatic facilitation? Separating sequential effects in two-choice reaction time. Journal of Experimental Psychology: Human Perception \& Performance, 11, 598-616.

Sommer, W., Leuthold, H., \& Soetens, E. (1999). Covert signs of expectancy in serial reaction time tasks revealed by event-related potentials. Perception \& Psychophysics, 61, 342-353.

Sommer, W., Matt, J., \& Leuthold, H. (1990). Consciousness of attention and expectancy as reflected in event-related potentials and reaction times. Journal of Experimental Psychology: Learning, Memory, \& Cognition, 16, 902-915.

VAN SELST, M., \& Jolicceur, P. (1994). A solution to the effect of sample size on outlier elimination. Quarterly Journal of Experimental Psychology, 47A, 631-650.

Winer, B. J. (1962). Statistical principles in experimental design. New York: McGraw-Hill.

Wolfe, J. M. (1998). Visual search. In H. Pashler (Ed.), Attention (pp. 13-73). Hove, U.K.: Psychology Press.

Woods, D. L., Alain, C., Diaz, R., Rhodes, D., \& Ogawa, K. H. (2001). Location and frequency cues in auditory selective attention. Journal of Experimental Psychology: Human Perception \& Performance, 27, 65-74.

Woods, D. L., \& Elmasian, R. (1986). The habituation of event-related potential to speech sounds and tones. Electroencephalography \& Clinical Neurophysiology, 76, 447-459.

\section{NOTES}

1. We thank Chris Darwin for this suggestion.

2. The value of this time delay $(2,100 \mathrm{msec})$ was selected in light of the results of Experiments $2 \mathrm{~A}$ and $2 \mathrm{~B}$, in which substantial repetition effects were observed at an RSI of $1,300 \mathrm{msec}$, and a conservative estimate of RT at $800 \mathrm{msec}$. Whereas average RT for Experiment 2 was $558 \mathrm{msec}$, we felt it necessary to include an additional overhead so that the majority of responses (if not all) could be initiated within the allotted time frame.

3. The unique indexing of FSHD contingencies at frontocentral N2, prior to the unique indexing of FSHS contingencies at frontocentral P3, may reflect the decision to include both incorrect and correct trials during ERP analysis, in the interests of achieving homogeneous signal-tonoise ratios between conditions.

(Manuscript received September 18, 2007; revision accepted for publication June 7, 2008.) 